\title{
Outcomes of Zika virus infection during pregnancy: contributions to the debate on the efficiency of cohort studies
}

\author{
Elisabeth Carmen Duarte ${ }^{1,2^{*}}$, Leila Posenato Garcia ${ }^{3,4}$, Wildo Navegantes de Araújo ${ }^{2,5}$ and Maria P. Velez ${ }^{6}$
}

\begin{abstract}
Background: Zika infection during pregnancy (ZIKVP) is known to be associated with adverse outcomes. Studies on this matter involve both rare outcomes and rare exposures and methodological choices are not straightforward. Cohort studies will surely offer more robust evidences, but their efficiency must be enhanced. We aim to contribute to the debate on sample selection strategies in cohort studies to assess outcomes associated with ZKVP.

Main body of the abstract: A study can be statistically more efficient than another if its estimates are more accurate (precise and valid), even if the studies involve the same number of subjects. Sample size and specific design strategies can enhance or impair the statistical efficiency of a study, depending on how the subjects are distributed in subgroups pertinent to the analysis. In most ZIKVP cohort studies to date there is an a priori identification of the source population (pregnant women, regardless of their exposure status) which is then sampled or included in its entirety (census). Subsequently, the group of pregnant women is classified according to exposure (presence or absence of ZIKVP), respecting the exposed: unexposed ratio in the source population. We propose that the sample selection be done from the a priori identification of groups of pregnant women exposed and unexposed to ZIKVP. This method will allow for an oversampling (even 100\%) of the pregnant women with ZKVP and a optimized sampling from the general population of pregnant women unexposed to ZIKVP, saving resources in the unexposed group and improving the expected number of incident cases (outcomes) overall.

Conclusion: We hope that this proposal will broaden the methodological debate on the improvement of statistical power and protocol harmonization of cohort studies that aim to evaluate the association between Zika infection during pregnancy and outcomes for the offspring, as well as those with similar objectives.
\end{abstract}

Keywords: Zika virus infection, Cohort studies, Efficiency, Pregnancy outcome, Congenital abnormalities, Epidemiologic studies

\section{Background}

Evidence to date indicates that infection by the Zika virus during pregnancy (ZKVP) is associated with the occurrence of microcephaly and other malformations of the central nervous system [1]. However, a large gap in knowledge still exists and further studies are needed to better understand this association. In this context experimental studies are ethically unviable, and analytical observational studies (cohort and case-control) are the only options available to test etiological and prognostic hypotheses [2].

\footnotetext{
* Correspondence: eduarte@unb.br

${ }^{1}$ Medical School, University of Brasilia- UnB, Brasilia, Brazil

${ }^{2}$ Núcleo de Medicina Tropical, Campus Universitário Darcy Ribeiro, Asa Norte, Caixa Postal 4517, Brasília, DF 70910-900, Brazil

Full list of author information is available at the end of the article
}

Approaches of the case-control type are more efficient when studying rare outcomes, but have a high potential for selection and differential misclassification biases. Cohort designs are more efficient when studying rare exposures, with the advantage of minimizing some biases. However, Zika infection during pregnancy involves both rare outcomes and rare exposures, and therefore, the methodological choices are not straightforward. Cohort studies will surely offer more robust evidences, but its efficiency must be enhanced. The objective of this article is to contribute to the debate on sample selection strategies in cohort studies to assess outcomes associated with ZKVP. 


\section{Discussion}

\section{Efficiency of epidemiological studies}

Rothman and Greenland (2008) define study efficiency as the relationship between the amount of information extracted from the data and the number of subjects recruited for the study, or the cost involved to acquire such data [2]. A study can be statistically more efficient than another if its estimates are more accurate (precise and valid), even if the studies involve the same number of subjects (and perhaps the same cost). "Cost" has a broad meaning here, including but not limited to the number of subjects involved in the study. Besides, sample size specific design strategies can enhance or impair the statistical efficiency of a study, depending on how the subjects are distributed in subgroups pertinent to the analysis.

Definition of the population(s) to be sampled in cohort studies In most ZKVP cohort studies to date (which follow the protocol for ZKVP studies recommended by the WHO [3]), when choosing groups for comparison (of exposed and unexposed subjects), there is an a priori identification of the source population (pregnant women, regardless of their exposure status) which is then sampled or included in its entirety (census). Subsequently, the group of pregnant women is classified according to exposure (presence or absence of ZKVP), respecting the expose$\mathrm{d}$ :unexposed ratio in the source population. Among the many advantages of this method, it allows a direct estimate of the risk of exposure to infection by the Zika virus (including both symptomatic and asymptomatic cases) in the general population of pregnant women (source population). However, this approach compromises the efficiency of a study considering the rareexposure context, since the required minimum sample size may be larger than the sample size required in other strategies to achieve the same (or even better) statistical power. For example, let's consider the following parameters: in a given population, $1.0 \%$ of pregnant women will be infected by the Zika virus (exposed group); among them, $5.0 \%$ will present the outcome of interest to the study (i.e., neurological changes in the conceptus), and $0.25 \%$ will present the outcome among the unexposed pregnant women. In total, it would require a minimum sample size of approximately 2,700 pregnant women to find a mere 27 exposed, and a total of eight outcomes (one among the exposed and seven among the unexposed mothers) to arrive at a statistical power of $59 \%$ and a type 1 error of $5 \%$ for crude analysis [4] (Table 1, example 1). Despite the large sample size, the small number of outcomes would greatly limit the efficiency of the study for stratified or multivariate analyses. It is clear that to better understand the natural history of ZKVP, more efficient studies are needed, including those supported by reliable data bases that allow testing for interactions and adjusting for confounding variables.

Aware of this limitation, we propose that the sample selection be done from the a priori identification of

Table 1 Different sample strategies and characteristics to assess outcomes associated with Zika infection during pregnancy in analytical cohort studies

\begin{tabular}{|c|c|c|c|}
\hline \multirow[t]{2}{*}{ Characteristics } & \multirow{2}{*}{$\begin{array}{l}\text { Sample of study } \\
\text { population } \\
\text { Example } 1\end{array}$} & \multicolumn{2}{|c|}{$\begin{array}{l}\text { Sample of unexposed pregnant women, and census } \\
\text { of exposed population (from study population). }\end{array}$} \\
\hline & & Example 2 & Example 3 \\
\hline $\begin{array}{l}\text { Study population size of eligible } \\
\text { pregnant women (Study population) }\end{array}$ & 20.000 & 20.000 & 20.000 \\
\hline Sample size & 2.700 & 800 (ratio 1:3) & 1.200 (ratio 1:5) \\
\hline Exposed (sample) & $27(1 \%)^{a}$ & & \\
\hline Unexposed (sample) & 2673 & & \\
\hline $\begin{array}{l}\text { Exposed (from population identified } \\
\text { by surveillance systems) }\end{array}$ & - & $200(1 \%)^{a}$ exposed & $200(1 \%)^{a}$ exposed \\
\hline $\begin{array}{l}\text { Unexposed (from population identified } \\
\text { by surveillance systems) }\end{array}$ & - & $600-6$ exposed $(1 \%)=594$ unexposed & $\begin{array}{l}1.000-10 \text { exposed }(1 \%)=990 \\
\text { unexposed }\end{array}$ \\
\hline Ratio exposed:unexposed & 1:99 (as the study population) & 1:3 (defined by investigator) & 1:5 (defined by investigator) \\
\hline $\begin{array}{l}\text { Expected cases among exposed } \\
\text { (rounded numbers) }\end{array}$ & $1(5 \%)^{a}$ & $10(5 \%)^{a}$ & $10(5 \%)^{a}$ \\
\hline $\begin{array}{l}\text { Expected cases among unexposed } \\
\text { (round numbers) }\end{array}$ & $7(0.25 \%)^{\mathrm{a}}$ & $1(0.25 \%)^{\mathrm{a}}$ & $2(0.25 \%)^{a}$ \\
\hline Total expected cases (round numbers) & 8 & 11 & 12 \\
\hline Type 1 error & 0.05 & 0.05 & 0.05 \\
\hline Study Power ${ }^{b}$ & $59 \%$ & $95 \%$ & $97 \%$ \\
\hline
\end{tabular}

aParameters: $1 \%$ of pregnant women will be infected by the Zika virus; 5 and $0.25 \%$ of infected and non-infected pregnant women will present the outcome, respectively

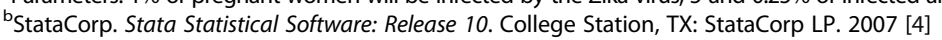


groups of pregnant women exposed and unexposed to ZKVP. This method will allow for an oversampling (even $100 \%$ ) of the pregnant women with ZKVP and a optimized sampling from the general population of pregnant women unexposed to ZKVP, saving resources in the unexposed group and improving the expected number of incident cases (outcomes) overall. In the context of nonassociation, a ratio of 1:1 between exposed and unexposed would be most efficient. Recognizing a priori that there is a known association and that exposure to ZKVP is a risk factor, the ratio should favor the group of unexposed mothers where fewer cases (outcomes) are expected. Thus, one could choose an optimized ratio for exposed and unexposed pregnant women of $1: 3$ or $1: 4$ or 1:5 [5] (Table 1, examples 2 and 3).

Considering the same parameters described above, a sample of only 1200 pregnant women (200 exposed and 1000 unexposed, 1:5 ratio) would give better results compared to those obtained in a sample more than two times as large, as in the strategy previously described. Approximately 12 outcome cases would be found, ten among exposed and two among unexposed pregnant women, ensuring a higher statistical power (97\%) in crude analyses than in the previous example [4] (Table 1, example 3). Table 1 shows expected results for these two sampling strategies.

We acknowledge the difficulty of identifying populations of ZKVP-exposed and unexposed pregnant women and the biases inherent in the selection of subjects based on secondary data. However, many countries, including Brazil, possess consolidated health information systems and have adopted surveillance protocols targeting women suspected of having ZKVP or other vertically communicable exanthematous diseases. In such countries, the following procedures may be helpful to identify exposed and unexposed pregnant women: i) an a priori identification of symptomatic pregnant women with suspected ZKVP through routine surveillance notification and of peers in the prenatal care services without suspected ZKVP; ii) posteriorly, to carry out laboratory tests to confirm or rule out the presence of ZKVP in both groups, allowing to recompose the exposed and unexposed groups; iii) subsequently, to follow-up the unexposed group (including lab tests) of women for the detection of exposure if it occurs at any time during the remaining gestational period, and again to recompose the exposed and unexposed groups. As an additional gain, detection bias by surveillance protocol can be estimated from the results of tests conducted on samples collected from the pregnant women unsuspected of having been exposed to ZKVP.

We also recognize the limitation of the currently available commercial laboratory test to accurately diagnose Zika virus infection during pregnancy, mainly in the asymptomatic cases with probable low viremia or antibody response. New tests are being developed, including the MAC-Elisa IgG and IgM test developed by the CDC to differentiate dengue fever infection and Zika infection by running both antigens together [6]. However, there is a need to develop rapid diagnosis tests to support health care professionals more efficiently when assisting pregnant women at risk of ZKVP.

\section{Conclusion}

We hope that this reflection will broaden the methodological debate on this subject. The sampling strategy we have proposed may improve the statistical power of studies that aim to evaluate the association between Zika infection during pregnancy and outcomes for the offspring, as well as those with similar objectives. Besides that, the harmonization of Zika virus research protocols is also desirable to obtain comparable data in order to allow evidence generation regarding very rare outcomes when individual study power will probably not be sufficient.

\section{Acknowledgements}

Not applicable.

Funding

We have no funding to declare.

Availability of data and materials

Not applicable.

Authors' contributions

ECD and LPG conceived the study, ECD elaborated the first drafted of the manuscript; all authors, ECD, LPG, WNA and MPV, contributed to the final document and critically revised the manuscript for intellectual content. All authors read and approved the final manuscript.

Ethics approval and consent to participate

Not applicable.

Consent for publication

Not applicable.

Competing interests

All coauthors have no conflicts of interest to declare.

\section{Publisher's Note}

Springer Nature remains neutral with regard to jurisdictional claims in published maps and institutional affiliations.

\section{Author details}

${ }^{1}$ Medical School, University of Brasilia- UnB, Brasília, Brazil. ${ }^{2}$ Núcleo de Medicina Tropical, Campus Universitário Darcy Ribeiro, Asa Norte, Caixa Postal 4517, Brasília, DF 70910-900, Brazil. ${ }^{3}$ Institute of Applied Economic Research - IPEA, Brasília, Brazil. ${ }^{4}$ Institute of Applied Economic Research SBS Quadra 1, Bloco J, Ed. BNDES/Ipea, Brasília, DF 70076-900, Brazil. ${ }^{5}$ Faculty of Ceilândia, University of Brasília - UnB, Brasília, Brazil. " 5 School of Medicine Department of Obstetrics and Gynaecology, Queen's University, 99 University Ave, Kingston, ON K7L 3N6, Canada. 
Received: 23 August 2017 Accepted: 15 November 2017

Published online: 02 December 2017

\section{References}

1. Awadh A, Chughtai AA, Sheikh M, Heslop DJ, Macintyre CR. Does Zika virus cause microcephaly - applying the Bradford Hill viewpoints. PLOS Currents. 2017;

2. Rothman KJ, Greenland S, Lash TL. Modern epidemiology. 3rd ed. Philadelphia: Lippincott-Wolters-Kluwer; 2008.

3. WHO. Harmonization of ZIKV Research Protocols to address key public health concerns. WHO/ Institut Pasteur Draft v4.0. 2017. [Internet]. 2017 June. Available from: http://www.who.int/reproductivehealth/zika/ZIKVProtocol-Summary-for-WHO-v4-2.pdf?ua=1.

4. STATACORP. Stata statistical software: release 10. College Station, TX: StataCorp LP; 2007.

5. Walter SD. Determination of significant relative risks and optimal sampling procedures in prospective and retrospective comparative studies of various sizes. Am J Epidemiol. 1977;105(4):387-97. in ROTHMAN KJ, GREENLAND S, LASH TL. Modern epidemiology. 3rd ed. Philadelphia: Lippincott-WoltersKluwer; 2008

6. WHO. Laboratory testing for Zika virus infection. Interim guidance. WHO/ ZIKV/LAB/16.1. 2016. [Internet]. 2017 June. Available from: http://www.who. int/csr/resources/publications/zika/laboratory-testing/en/.

Submit your next manuscript to BioMed Central and we will help you at every step:

- We accept pre-submission inquiries

- Our selector tool helps you to find the most relevant journal

- We provide round the clock customer support

- Convenient online submission

- Thorough peer review

- Inclusion in PubMed and all major indexing services

- Maximum visibility for your research

Submit your manuscript at www.biomedcentral.com/submit
Biomed Central 